\title{
CONDITIONS FOR THE ABSOLUTE CONTINUITY OF TWO DIFFUSIONS
}

\author{
BY
}

\author{
STEVEN OREY
}

ABSTRACT. Consider two diffusion processes on the line. For each starting point $x$ and each finite time $t$, consider the measures these processes induce in the space of continuous functions on $[0, t]$. Necessary and sufficient conditions on the generators are found for the induced measures to be mutually absolutely continuous for each $x$ and $t$. If the first process is Brownian motion, the second one must be Brownian motion with drift $b(x)$, where $b(x)$ is locally in $L_{2}$ and satisfies a certain growth condition at $\pm \infty$.

0. Introduction. Our concern is with diffusion processes on an open one -dimensional interval $l$, having homogeneous transition probabilities, and possessing no singular points. We do not allow curtailment of life time (killing), and the end points of $I$ must be inaccessible. This class of diffusions will be denoted by $\mathscr{D}$, or $D(I)$ if the dependence on $I$ needs to be indicated. A standard way of realizing such a diffusion is via coordinate space: $C$ is to be the class of all continuous functions from $[0, \infty)$ into $I$, and for $\omega \in C$ let $X_{t}(\omega)=\omega(t)$. Let $C_{t}$ be the $\sigma$-field generated by $\left\{X_{s}: s \leq t\right\}$, and $\mathcal{C}$ the least $\sigma$-field including all the $\mathcal{C}_{t}, 0 \leq t<\infty$. A diffusion in $\mathcal{D}$ is then given by a collection $P=\left(P_{x}\right), x \in I$, of probability measures on $(C, \mathcal{C})$; (for details see $[7, \mathrm{p} .84]$, or $[4, \mathrm{p} .102]$ ). We let $\left.P_{x}\right|_{t}$ be the restriction of $P_{x}$ to $C_{t}$. Given two diffusions $P^{1}$ and $P^{2}$ in $D, P^{1} \prec^{x} P^{2}$ is to mean that $\left.\left.P_{x}^{1}\right|_{t} \ll P_{x}^{2}\right|_{t}$ for each $x \in I, 0 \leq t<\infty$, where $\ll$ means "is absolutely continuous with respect to". Now each $P \in D$ is determined by a scale function $p$ and a speed measure $m$; we write $P \sim(p, m)$. In $\$ 2$ we give necessary and sufficient conditions for $P^{1} \prec P^{2}$ in terms of the associated scales and speed measures. The special case $I=(-\infty, \infty)$ and $P^{2}$ Wiener measure is discussed in $\$ 1$. It turns out that in this case $P^{1}$ must correspond to Brownian motion with a suitable drift: the condition on the drift coefficient $b(x)$ is that it is locally square integrable and satisfies a certain growth condition at $\pm \infty$; the growth condition is simply the one dictated by the inaccessibility of the end points. It is also shown that the conditions on $b(x)$ are necessary and sufficient conditions

Received by the editors October 4, 1973.

AMS (MOS) subject classifications (1970). Primary 60J60, 28A40. 
for the process $\exp \left[\int_{0}^{t} b\left(X_{u}\right) d X_{u}-1 / 2 \int_{0}^{t} b^{2}\left(X_{u}\right) d u\right]$ to be a martingale under $P^{2}$, i.e. Wiener measure.

We proceed to some notational points and details. In place of the speed measure we will usually deal with the associated distribution function: $m(x)=$ $m((-\infty, x])$. If $P \sim(p, m)$ then also $P \sim\left(p^{*}, m^{*}\right)$, where $p^{*}(x)=a p(x)+b$, $m^{*}(x)=a^{-1} m(x)+c$, where $a$ is a positive number, $b$ and $c$ arbitrary; but, except for this trivial kind of nonuniqueness, $(p, m)$ is determined by $P$. Conversely $(p, m)$ determines $P$. We recall that any continuous strictly increasing function $p(x)$ can serve as a scale, while the speed measure is a positive measure, finite on compact sets, strictly positive on open sets. The assumption that the end points are inaccessible imposes an additional condition. For the case $I=$ $(-\infty, \infty)$ this is

$$
\int_{c}^{\infty}(p(\infty)-p(y))_{m}(d y)=\int_{-\infty}^{c}(p(y)-p(-\infty))_{m}(d y)=\infty, \quad-\infty<c<\infty
$$

where $p(\infty)$ and $p(-\infty)$ denote the obvious limits. The condition is due to Feller [3]; in his terminology $+\infty$ and $-\infty$ are not exit boundaries. For details consult [1] or [7].

On the space $C$ shift operators $\theta_{t}$ are defined in the natural way: $\left(\theta_{t} \omega\right)(s)=$ $\omega(t+s)$. If $X$ is a diffusion in $D_{I}, B$ a Borel subset of $l$, we write $\mu(t, B ; X)=$ $\int_{0}^{t} \chi_{B}\left(X_{s}\right) d s$. Thus $\mu(t, B ; X)$ is the sojourn time of $X$ in $B$ up to time $t$. For fixed $t$ this is a measure on the Borel sets of $l$. It is known to be absolutely continuous with respect to the speed measure $m$ of $X$, and there exists a nice version of the Radon-Nikodym derivative, known as the local time: thus $\mu(t, B ; X)=$ $\int_{B} L(t, x ; X) m(d x)$. Here for fixed $t$ and $x, L(t, x ; X)$ is a random variable; and for fixed $\omega,(t, x) \rightarrow L(t, x ; X)$ is, with probability one, continuous. Further details and references about this and other matters needed in the body of the paper are collected in the appendix.

Whenever dealing with $P$, possibly with affixes, we will use $E$ with the same affixes to denote the expectation operator corresponding to the probability measure denoted by $P$.

1. Brownian motion with drift. Throughout this section $\mathscr{D}=\mathscr{D}_{(-\infty, \infty)}$, and $P^{0}=\left(P_{x}^{0}\right),-\infty<x<\infty$, is the element of $\mathscr{D}$ corresponding to the Wiener distribution; so the coordinate process $\left(X_{t}\right)$ is Brownian motion under $P^{0}$. Somewhat more generally, if $Z=\left(Z_{t}\right), 0 \leq t<\infty$, is a real-valued stochastic process on $C$, and $\left(P_{x}\right) \in \mathscr{D}$, and (a) $Z_{t}$ is $\mathcal{C}_{t}$-measurable for each $t$, (b) the finite-dimensional distributions of the $Z$ process under $P_{x}$ agree with the finite-dimensional distributions of the coordinate process $X$ under $P_{x}^{0}$ for each $x$, (c) $E\left[Z_{t} \mid \mathcal{C}_{s}\right]=Z_{s}$ 
$P_{x}$ a.s. for $0 \leq s<t,-\infty<x<\infty$, then $Z$ will be said to be a Brownian motion with respect to $\left(P_{x}\right)$.

Suppose $P^{\prime} \in \mathcal{D}, P^{\prime} \prec P^{0}$. For each $x$ and $t$ let $L_{t}^{(x)}$ be the Radon-Nikodym derivative of $\left.P_{x}^{\prime}\right|_{t}$ with respect to $\left.P_{x}^{0}\right|_{t}$. Then, as discussed in III of the appendix, $\left(L_{t}^{(x)}, C_{t}, t \geq 0\right)$ is a martingale under $P_{x}^{0}$, and we can choose a right continuous version. Note that $L_{0}^{(x)}=1 P_{x}^{0}$-a.s. by the zero-one law. $L_{t}^{(x)}$ is Borel measurable in $x$; this can be seen by expressing the Radon-Nikodym derivative explicitly as a limit of difference quotients. We may then define: $L_{t}=L_{t}^{\left(X_{0}\right)}$. Thus

$$
\left.d P_{x}^{\prime}\right|_{t} /\left.d P_{x}^{0}\right|_{t}=L, \quad 0 \leq t<\infty,-\infty<x<\infty
$$

By $L_{\text {loc }}^{1}\left(L_{\text {loc }}^{2}\right)$ we mean the class of Borel measurable functions $b(x)$ defined on $(-\infty, \infty)$ which are integrable (square integrable) over compact intervals. We will use the notation

$$
L_{t}[b]=\exp \left\{\int_{0}^{t} b\left(X_{u}\right) d X_{u}-\frac{1}{2} \int_{0}^{t} b^{2}\left(X_{u}\right) d u\right\}, \quad 0 \leq t<\infty, b \in L_{\text {loc }}^{2}
$$

here $X$ will be coordinate process under Wiener measure. For the existence of the integrals see Appendix (I.C). We also will use

$$
Y_{t}[b]=X_{t}-\int_{0}^{t} b\left(X_{u}\right) d u, \quad 0 \leq t<\infty, b \in L_{\text {loc }}^{1}
$$

We write $L[b]$ or $Y[b]$ for the process $\left(L_{t}[b], \mathcal{C}_{t}, t \geq 0\right)$, respectively $\left(Y_{t}[b], \mathcal{C}_{t}\right.$, $t \geq 0$ ).

Our first proposition is a Markov process variant of a result of Kailath and Zakai [10]; parts of the argument trace back to Hitsuda [6].

Proposition 1. Let $P^{\prime} \in \mathfrak{D}, P^{\prime} \prec P^{0}$. Then there exists $b \in L_{\text {loc }}^{2}$ such that

$$
\left.d P_{x}^{\prime}\right|_{t} /\left.d P_{x}^{0}\right|_{t}=L[b], \quad 0 \leq t<\infty,-\infty<x<\infty .
$$

It follows that

$$
\begin{aligned}
& E_{x} L_{t}[b]=1, \quad 0 \leq t<\infty,-\infty<x<\infty . \\
& Y[b] \text { is a Brownian motion under } P^{\prime} .
\end{aligned}
$$

Remark 1. For any $P^{\prime} \in \mathcal{D}$ condition (1.6) can be satisfied for at most one function $b$, where we identify two functions which are equal a.e. For otherwise, one would obtain the difference of two Brownian motions, i.e. two continuous martingales, represented as a function of bounded variation, which is impossible 
except in the trivial case where the function of bounded variation vanishes identically.

Proof. Obtain a right continuous martingale $\left(L_{t}\right)$ satisfying $(1.1)$ as above. It must be shown that $\left(L_{t}\right)$ is a multiplicative functional of Brownian motion. Let $H$ be a bounded $C_{t}$-measurable random variable. One obtains easily (this is an instance of (3.1)) that

$$
E_{x}^{\prime}\left[H \circ \theta_{s} \mid \mathcal{C}_{s}\right]=E_{x}\left[\left(H \circ \theta_{s} \cdot L_{t+s} / L_{s}\right) \mid \mathcal{C}_{s}\right], \quad P_{x}^{\prime} \text {-a.s. }
$$

Also, using the Markov property of $P^{0}$,

$$
E_{X}^{\prime}[H]=E_{X}^{0}\left[H L_{t}\right]=E_{x}\left[H \circ \theta_{s} \cdot L_{t} \circ \theta_{s} \mid C_{s}\right], \quad P_{x}^{0} \text {-a.s. }
$$

By the Markov property of $P^{\prime}$, the first members of $(1.7)$ and $(1.8)$ agree $P_{x}^{\prime}$-a.s. Since $P^{\prime} \prec P^{0}$ we can conclude that the last members of $(1.7)$ and $(1.8)$ agree $P_{x}^{\prime}$-a.s.; and the exceptional set $\Lambda$ on which agreement fails belongs to $C_{s}$, $P_{x}^{\prime}[\Lambda]=0$. Throughout this discussion $x$ is arbitrary but fixed. Keeping $(1.1)$ in mind, we may infer that $P_{x}^{0}\left[\Lambda \cap\left[L_{s}>0\right]\right]=0$. Since every $\mathcal{C}_{s+t}$-measurable random variable is of the form $H \circ \theta_{s}$ for some $\mathcal{C}_{t}$-measurable $H$, it follows that $L_{t+s}=L_{s} \cdot L_{t} \circ \theta_{s} P_{x}^{0}$-a.s., for, by what has been said already, the equality holds $P_{x}^{0-a . s . ~ o n ~ t h e ~ s e t ~}\left[L_{s}>0\right]$; and, as already remarked, if $L_{s}=0$, then $P_{x}^{0}$ a.s. also $L_{t+s}=0$. So $\left(L_{t}\right)$ is a multiplicative functional of Brownian motion. As already noted, $L_{0}=1$, and $\left(L_{t}\right)$ is a martingale with respect to the $\sigma$-fields $\mathcal{C}_{t}$ generated by our Brownian motion (coordinate process). It follows that one has a representation $L_{t}-1=\int_{0}^{t} H_{u} d X$ where $H_{u}$ is $C_{u}$ measurable and $\int_{0}^{t} H_{u}^{2} d u<\infty P_{x}^{0}$ a.s. Indeed, if $L_{t}$ is square integrable, such a representation is known to hold with $E\left[\int_{0}^{t} H_{u}^{2} d u\right]<\infty$ (see Kunita-Watanabe [11] or Meyer [12]) and, as pointed out by Hitsuda [6], an easy argument using stopping times gives the result needed here. In particular, then, $L_{t}$ is continuous. Let $A_{t}=-\log L_{t}$. This gives rise to an additive functional, with $A_{0}=0 P_{x}$-a.s. The values of $A_{t}$ lie in $(-\infty, \infty]$, but $A_{t}$ is continuous in the topology of the extended line. It follows (see Appendix II) that $A$ must actually be finite valued. That is, $L_{t}>0$ and we may apply Ito's formula to obtain

$$
\log L_{t}=\int_{0}^{t} \frac{1}{L_{s}} d L_{s}-\frac{1}{2} \int_{0}^{t} \frac{H_{s}^{2}}{L_{s}^{2}} d s .
$$

The first term on the right is a continuous local martingale; it is also an additive functional, because $\left(L_{t}\right)$ is a multiplicative functional. We now apply Tanaka's representation theorem to this term (see Appendix II), obtaining 


$$
\int_{0}^{t} \frac{1}{L_{s}} d L_{s}=\int_{0}^{t} k\left(X_{s}\right) d X_{s}+g\left(X_{t}\right)-g\left(X_{0}\right)
$$

with $k \in L_{\text {loc }}^{2} g$ a continuous function. According to Tanaka, if $J$ is any compact interval, $\tau=\inf \left\{t: X_{t} \notin J\right\}$, each of the terms of (1.9) when evaluated at $t \wedge \tau$ has finite moments of all orders. Therefore, the two stochastic integrals evaluated at $t \wedge r$ define martingales; then $g\left(X_{t \wedge r}\right)$ is a martingale. As a consequence (see Dynkin [2, Theorem 13.10]) $g$ is harmonic, i.e. $g(x)=a x+c$. Obviously we may set $c=0$, and letting $b(x)=k(x)+a$ gives $\int_{0}^{t}\left(1 / L_{s}\right) d L_{s}=\int_{0}^{t} b\left(X_{s}\right) d X_{s}$.

Let $M_{t}$ denote the first term of (1.9). The continuous local martingale $\left(M_{t}\right)$ has an associated continuous increasing process $\langle M, M\rangle$ (notation as in [11] or [12]) satisfying

$$
\langle M, M\rangle_{t}=\int_{0}^{t} \frac{H_{s}^{2}}{L_{s}^{2}} d s=\int_{0}^{t} b^{2}\left(X_{s}\right) d s
$$

and (1.4) is established. Thus $L_{t}=L_{t}[b] P_{x}^{0}$-a.s., and (1.5) follows immediately. Finally (1.6) is an instance of Girsanov's theorem (see Appendix III).

For a diffusion belonging to $\mathcal{D}$ with differential generator $1 / 2\left(d^{2} / d x^{2}\right)+$ $d b(x) / d x$ with $b$ bounded and continuous, one checks easily that the scale and speed are given by

$$
p_{b}(x)=\int_{0}^{x} \exp \left\{-2 \int_{0}^{y} b(z) d z\right\} d y, \quad m_{b}(x)=2 \int_{0}^{x} \exp \left\{2 \int_{0}^{y} b(z) d z\right\} d y .
$$

These expressions make sense whenever $b \in L_{\text {loc }}^{1}$. For $\left(p_{b}, m_{b}\right)$ to correspond to some diffusion in $\mathcal{D}$ one needs, in addition, the inaccessibility condition (0.1), which now takes the form

$$
\int_{c}^{\infty}\left(\frac{1}{\beta(y)} \int_{y}^{\infty} \beta(u) d u\right) d y=\int_{-\infty}^{c}\left(\frac{1}{\beta(y)} \int_{-\infty}^{y} \beta(u) d u\right) d y=\infty, \quad-\infty<c<\infty,
$$

where $\beta(y)=\exp \left\{-2 \int_{0}^{y} b(z) d z\right\}$.

Proposition 2. Let $P^{\prime} \in \mathcal{D}, P^{\prime} \sim\left(p_{b}, m_{b}\right)$, where $b \in L_{\text {loc }}^{1}$. Then (1.6) bolds.

Proof. If $b$ is bounded and Lipschitz continuous this is known. Indeed $P^{\prime}$ is then determined by its differential generator $d^{2} / d x^{2}+d b(x) / d x$. On the other hand, a diffusion with this differential generator can be obtained as a solution of the Ito stochastic integral equation $Z_{t}=X_{t}+\int_{0}^{t} b\left(Z_{u}\right) d u, P_{x}^{0-a . s \text {. and (1.6) }}$ follows.

In the general case choose a sequence $b_{n}$ of bounded Lipschitz continuous functions converging to $b$ in the $L^{1}$-sense on compact intervals. Write $b_{\infty}$ for $b$, and $p_{n}, m_{n}$ for $p_{b_{n}}, m_{b_{n}}$ respectively, $n=1,2, \cdots, \infty$. Now, under $P^{0},\left(X_{t}\right)$ is 
Brownian motion, and diffusions $Z^{(n)}$ with scale $p_{n}$ and speed $m_{n}$ can be realized (see Appendix I) as

$$
Z_{t}^{(n)}=p_{n}^{-}\left(X_{A_{t}^{(n)}}\right), \quad n=1,2, \cdots, \infty,
$$

where $p_{n}^{*}$ is the inverse function of $p_{n}$, and $A_{t}^{(n)}$, as a function of $t$, is the inverse of $\tau_{t}^{(n)}$, where $\tau_{t}^{(n)}=\int_{-\infty}^{\infty} L(t, x ; X) m_{n}(d x)$. Writing $Y_{t}^{(n)}=Z_{t}^{(n)}-$ $\int_{0}^{t} b_{n}\left(Z_{s}^{(n)}\right) d s$, we know $\left(Y_{t}^{(n)}\right)$ is Brownian motion under $P^{0}$ for $n=1,2, \cdots$. We wish to prove the same assertion for $n=\infty$ by a limiting argument. Indeed $Y_{t}^{(n)}$ approaches $Y_{t}^{(\infty)}$ in a very strong sense: With probability one convergence holds for all $t$, uniformly for $t$ in any compact interval. To see this recall some properties of $L(t, x ; X)$ : It is continuous in $(t, x)$, nondecreasing in $t$, and, for fixed $t$, vanishes outside some finite $x$-interval. One then verifies easily that a.s.

$A_{t}^{(n)}$ converges to $A_{t}^{(\infty)}$ for all $t$, uniformly for $t$ in any compact set. Also $p_{n}^{-}(x)$ converges to $p_{\infty}^{-}(x)$ uniformly for $x$ in any compact subset of $(p(-\infty), p(\infty))$. Therefore a.s. $Z_{t}^{(n)}$ converges to $Z_{t}^{(\infty)}$, uniformly for $t$ in any compact interval. One also obtains (see Appendix (I.C)) that a.s.

$$
\begin{aligned}
\int_{0}^{t} b_{n}\left(Z_{s}^{(n)}\right) d s= & \int_{-\infty}^{\infty} L\left(A_{t}^{(n)} p_{n}^{-}(x) ; X\right) b_{n}(x) m_{n}(d x) \\
& \rightarrow \int_{-\infty}^{\infty} L\left(A_{t}, p^{-}(x) ; X\right) b(x) m_{(}(d x)=\int_{0}^{t} b\left(Z_{s}\right) d s,
\end{aligned}
$$

the convergence being uniform for $t$ in any compact interval.

Proposition 3. Let $P^{\prime} \in \mathcal{D}, P^{\prime} \sim\left(p_{b}, m_{b}\right)$ for some $b \in L_{\text {loc }}^{2}$ Then $P^{\prime}<P^{0}$ and (1.4) bolds.

Proof. By Proposition 2, (1.6) holds. So under $P^{\prime}, X_{t}$ differs from the Brownian motion $Y_{t}[b]$ by $\int_{0}^{t} b\left(X_{u}\right) d u$. So $P^{\prime}<P^{0}$ follows as soon as $\int_{0}^{t} b^{2}\left(X_{u}\right) d u<\infty P_{x}^{t}$-a.s. is established (see Appendix III, Corollary). The fact that the integral is finite becomes obvious on writing

$$
\int_{0}^{t} b^{2}\left(X_{u}\right) d u=\int_{-\infty}^{\infty} L(t, x ; X) b^{2}(x) m_{b}(d x)
$$

(see Appendix (I.C)), remembering the nature of $L(t, x ; X), m_{b}(d x)$, and that $b \in L_{\text {loc }}^{2}$. So $P^{\prime} \prec P^{0}$ is established. Finally (1.4) follows from applying Proposition 1; the fact that the $b$ supplied by that proposition agrees with the one we started out with here is an immediate consequence of the uniqueness assertion contained in Remark 1.

Proposition 4. Let $P^{\prime} \in \mathcal{D}, P^{\prime} \prec P^{0}$. Then there exists a $b \in L_{\text {loc }}^{2}$ with $P^{\prime} \sim\left(p_{b}, m_{b}\right)$. 
Proof. Proposition 1 applies and supplies a unique $b \in L_{\text {loc }}^{2}$. We wish to conclude that $b$ satisfies the inaccess ibility condition (1.10). Let $r_{n}=$ $\inf \left\{t:\left|X_{t}\right|>n\right\}$ and let $b_{n}(x)=b(x)$ for $|x| \leq n+1, b_{n}(x)=0$ for $|x|>n+1$. Then $b_{n} \in L_{\text {loc }}^{2}$ and satisfies (1.10). So there exists $P^{(n)} \in \mathcal{D}, P^{(n)} \sim\left(p_{b_{n}}, m_{b_{n}}\right)$. Now $P^{(n)} \prec P^{(0)}$ and, using Proposition 1, we find

$$
\left.d P_{x}^{(n)}\right|_{\tau_{n}} /\left.d P_{x}^{0}\right|_{\tau_{n}}=\left.d P_{x}^{0}\right|_{\tau_{n}} /\left.d P_{x}^{0}\right|_{\tau_{n}}=L_{\tau_{n}}[b] .
$$

So $P^{\prime} \sim(p, m)$ with $p$ and $m$ agree ing with $p_{n}$ and $m_{n}$, respectively, on $[-n, n]$. Since $n$ is arbitrary $b$ must satisfy $(1.10)$, for otherwise $P_{x}^{\prime}\left[\sup _{s \leq t}\left|X_{s}\right|=\infty\right]>0$ for some finite $t$, contradicting $P^{\prime}<P^{0}$. In fact then $P^{\prime} \sim\left(p_{b}, m_{b}\right)$.

Theorem 1. For $P^{\prime} \in \mathcal{D}$ the following conditions are equivalent.

(a) $P^{\prime}<P^{0}$.

(b) $P^{\prime} \sim\left(p_{b}, m_{b}\right)$ for some $b \in L_{\text {loc }}^{2}$.

(c) $Y[b]$ is Brownian motion under $P^{\prime}$ for some $b \in L_{\text {loc }}^{2}$

(d) $P^{\prime} \sim(p, m)$, where $p$ bas an absolutely continuous, strictly positive derivative $p^{\prime}, m$ bas a derivative $m^{\prime}$ satisfying $1 / 2 p^{\prime}(x) m^{\prime}(x) \equiv 1$, and $p^{\prime \prime} \in L_{\text {loc }}^{2}$.

Proof. The equivalence of (a)-(c) follows from Propositions 1-4. Assuming (b), (d) follows at once; note that $b(x)=-1 / 2 p^{\prime \prime}(x) / p^{\prime}(x)$, and, since $p^{\prime}$ is strictly positive and continuous, the assumption $b \in L_{\text {loc }}^{2}$ gives $p^{\prime \prime} \in L_{\text {loc }}^{2}$. Similarly one can go from (d) to (b).

Corollary. $P^{\prime} \prec P^{0}$ implies $P^{0} \prec P^{\prime}$.

Proof. Note that the Radon-Nikodym derivative given in (1.4) is positive. $\$ 2$.

Remark 2. Also $P^{0} \prec P^{\prime}$ implies $P^{\prime} \prec P^{0}$; this follows from the result in

Here is an interesting consequence of Theorem 1. For $b \in L_{\text {loc }}^{2}$ and any $x$, $L_{t}[b]$ is always a supermartingale; it is a martingale if and only if $E_{x} L_{t}[b]=1$ for all $t$ (see Appendix III). We now have necessary and sufficient conditions for this.

Corollary 2. Let $b \in L_{10 c}^{2}$. If $b$ satisfies $(1.10), E_{x}^{0}\left[L_{t}[b]\right]=1$ for all $x$ and t. Conversely, if for some $x, E_{x}^{0}\left[L_{t}[b]\right]=1$ for all $t$, then $b$ satisfies $(1.10)$.

Proof. If $b$ satisfies (1.10), let $P^{\prime} \sim\left(p_{b}, m_{b}\right)$ and use (1.5) of Proposition 1. Suppose now that, for some $x, E_{x}^{0}\left[L_{t}[b]\right]=1$ for all $t$; a simple stopping time argument shows that this relation must then hold for all $x$, and we may use relation (1.4) to define the measures $P_{x}^{\prime}$. It follows easily (see Appendix III, transformation theorem) that $P^{\prime}=\left(P_{x}^{\prime}\right) \in \mathfrak{D}$. By Propositions 4, 2, 1 and Remark 1, $P^{\prime} \sim\left(p_{b}, m_{b}\right)$, which means that $b$ must satisfy $(1.10)$. 
2. The general case. Let $X^{i}=\left(X_{t}, \mathcal{C}_{t}, t \geq 0, P^{i}\right), i=1,2$, be the function space representation of two diffusions in $\mathscr{D}_{(-\infty, \infty)}$, with $P^{i}=\left(P_{x}^{i}\right)$. Let $P^{i} \sim$ $\left(p_{i}, m_{i}\right), i=1,2$. Applying $p_{1}$ to the coordinate process we obtain two new processes. Say $X^{i}=\left(Y_{t}, \mathcal{C}_{t}^{l}, t \geq 0, P^{i}\right), i=3,4$, where $Y_{t}=p_{1}\left(X_{t}\right)$, so that $\left(Y_{t}\right)$ is the coordinate process on the space $\mathcal{C}^{l}$ of continuous functions with values in $I=\left(p_{1}(-\infty), p_{1}(\infty)\right), \mathcal{C}_{t}^{I}$ is the $\sigma$-field generated by $\left\{Y_{s}: 0 \leq s \leq t\right\}$, and $P^{3}$ and $P^{4}$ are the measures on $C^{I}$ that are induced from $P^{1}$ and $P^{2}$, respectively, by the mapping $p_{1}$. Of course $X^{3}$ and $X^{4}$ are just $X^{1}$ and $X^{2}$ with the state space reparametrized, and the condition $P^{1}<P^{2}\left(P^{2}<P^{1}\right)$ is equivalent to $P^{3} \prec P^{4}\left(P^{4}<P^{3}\right)$.

Let $q$ be the inverse function of $p_{1}$. Then $P^{3} \sim\left(p_{3}, m_{3}\right)=\left(p_{1} \circ q, m_{3} \circ q\right)$, $P^{4} \sim\left(p_{4}, m_{4}\right)=\left(p_{2} \circ q, m_{3} \circ q\right)$. Note $X^{3}$ has Lebesgue scale on its interval of definition $I$.

We now make a time change $\beta(t)$ such that under $P^{3} Y_{\beta(t)}$ is a Brownian motion, defined up to first exit from $I$. Such a $\beta(t)$ is the inverse of the following additive functional on $X^{3}$ (see Appendix (I.D)),

$$
\alpha(t)=\int_{I} L\left(t, x ; X^{3}\right) 2 d x
$$

since $2 d x$ is the speed measure of Brownian motion.

Now we observe that if $P^{4} \prec P^{3}$ then $m_{4}$ and $m_{3}$ must be equivalent, that is, have the same null sets. Indeed one sees easily that $P_{x}^{i}\left[\mu\left(t, B ; X^{i}\right)>0\right]=0$ for every $t$ if and only if $m_{i}(B)=0$ (see Appendix I). So, if $P^{4}<P^{3}$ and $m_{3}(B)=$ 0 , then also $m_{4}(B)=0$. For the converse implication, suppose $m_{3}(B)>0$. Then $P_{x}^{3}\left[\mu\left(t, B ; X^{3}\right)>0\right]$ is positive for some $x$ and $t$. Also, for each $x$, $P_{x}^{3}\left[\mu\left(t, B ; X^{3}\right)>0\right.$ for all $\left.t\right]$ must equal 0 or 1 by the zero-one law. By considering $T=\inf \left\{t: \mu\left(t, B ; X^{3}\right)>0\right\}$ and using the strong Markov property we obtain the existence of some $x$ with $P_{x}^{3}\left[\mu\left(t, B ; X^{3}\right)>0\right.$ for all $\left.t\right]=1$. Then $P^{4} \prec P^{3}$ implies also $m_{4}(B)>0$.

The transformation taking $Y_{t}$ into $Y_{\beta(t)}$ transformed $X^{3}$ into Brownian motion defined up to leaving $l$. What process is obtained by applying the same transformation to $X^{4}$ ? To see this, recall the definition of local time to write

$$
\alpha(t)=\int_{1} \frac{d \mu\left(t, \cdot ; X^{3}\right)}{d m_{3}}(x) \cdot 2 d x .
$$

Interpret the indicated derivative as the limit superior of the difference quotients ordinarily defining a derivative. Because of the continuity of local time we know that $P_{x^{\prime}}^{3}$-a.s., for any $x^{\prime}$, this limit superior will actually be a limit for all $t$ and 
$x$. Assume now that $m_{3}$ and $m_{4}$ are equivalent. To consider $\alpha$ as a functional on $X^{4}$ write

$$
\alpha(t)=\int_{I} \frac{d \mu\left(t, \cdot ; X^{4}\right)}{d m_{3}}(x) \cdot 2 d x=\int_{I} \frac{d \mu\left(t, \cdot ; X^{4}\right)}{d m_{4}}(x) \frac{d m_{4}}{d m_{3}}(x) \cdot 2 d x .
$$

(Note that both $X^{3}$ and $X^{4}$ are coordinate processes, so $\mu\left(t, B ; X^{3}\right)$ and $\mu\left(t, B ; X^{4}\right)$ are two names for the same quantity.) If $t>0$, the derivative in the first integrand will exist as a limit of difference quotients and be positive $P_{x}^{3}$ a.s.; the same applies to the first derivative in the second integrand $P_{x}^{4}$-a.s. So, unless $P_{x}^{3}$ and $P_{x}^{4}$ are singular, the second derivative in the second integrand, which is not random, must also exist as a limit of difference quotients. This will be true always if $P^{3}<P^{4}$ or $P^{4}<P^{3}$. Then we may write

$$
\alpha(t)=\int_{1} L\left(t, x ; X^{4}\right) \frac{d m_{4}}{d m_{3}}(x) \cdot 2 d x .
$$

Now let

$$
X^{5}=\left(Y_{\beta(t)}, \mathcal{C}_{\beta(t)}^{I}, 0 \leq t<\alpha(\infty) ; P^{3}\right) \text { and } X^{6}=\left(Y_{\beta(t)}, \mathcal{C}_{\beta(t)}^{I}, 0 \leq t<\alpha(\infty), P^{4}\right)
$$

We know already that $X^{5}$ is a diffusion on $I$, defined up to the first exit time from $I$, with scale $x$ and speed $2 d x$. From the final form of $\alpha(t)$ we learn that (see Appendix (I.D)) $X^{6}$ corresponds to a diffusion with scale $p_{6}$ and speed $m_{6}$ given by

$$
p_{6}(x)=p_{4}(x), \quad m_{6}(d x)=2 \frac{d m_{4}}{d m_{3}}(x) d x
$$

defined on 1 . Since $X^{5}$ and $X^{6}$ have life times $a(\infty)$ which need not be infinite they do not necessarily belong to $D_{I}$, strictly speaking. However, $X^{5}$ and $X^{6}$ induce measures $P^{5}=\left(P_{x}^{5}\right)$ and $P^{6}=\left(P_{x}^{6}\right)$ on the space of all continuous functions from $[0, \infty)$ into $l$, defined up to the first time that the function approaches a boundary point of $I$. The measures $P^{5}, P^{6}$ come from the original measures $P^{3}, P^{4}$, via the map taking $\omega$ into $\beta[\omega]$, where $\beta[\omega](t)=\omega(\beta(t, \omega))$. If $\eta=\beta[\omega]$, $\omega(t)=\eta(\alpha(t, \omega))$, since $\alpha$ is the inverse of $\beta$. However, $\alpha$ can be considered as a function of $\eta$, because

$$
\int L\left(t, x ; X^{5}\right) m^{3}(d x)=\int L\left(\beta(t), x ; X^{3}\right) m^{3}(d x)=\beta(t)
$$

(see Appendix (I.C)), so that on a set having $P_{x}^{3}$-measure one for all $x$, the map $\omega$ into $\beta[\omega]$ is invertible. Observe now that for $P^{i}$ and $P^{j} \in \mathscr{D}_{P^{\prime}}, P^{i}<P^{i}$ if and only if $\left.\left.P_{x}^{i}\right|_{\tau} \ll P_{x}^{j}\right|_{\tau}$ for every $x \in I$, and every $\tau$ which is the first exit time from a 
compact subinterval of $I$, where $\left.\right|_{\tau}$ denotes restriction to $\mathcal{C}_{\tau}$. Similarly we can define $P^{6}<P^{5}\left(P^{5} \prec P^{6}\right)$ to hold if the measures are absolutely continuous when restricted up to the first exit time from any compact subinterval of 1 . From what we have said it follows that $P^{4}<P^{3}$ if and only if $P^{6}<P^{5}$, and both $P^{4}<P^{3}$ and $P^{3} \prec P^{4}$ if and only if both $P^{6} \prec P^{5}$ and $P^{5}<P^{6}$. Since $P^{5}$ is Brownian motion, defined up to the first exit time from $I$, we can use the work of \$1.

Theorem 2. $P^{2} \prec P^{1}$ implies $P^{1} \prec P^{2}$. Necessary and sufficient conditions for $P^{2}<P^{1}$ are as follows:

(i) the derivative $d p_{2}(x) / d p_{1}$ exists everywhere and defines a positive function absolutely continuous with respect to $p_{1}$;

(ii) $d m_{2}(x) / d m_{1}$ exists everywhere and satisfies $d m_{2}(x) / d m_{1} \cdot d p_{2}(x) / d p_{1}=1$;

(iii) the second derivative $d^{2} p_{2}(x) / d p_{1}^{2}$, defined $d p_{1}$-a.e. belongs to $L_{10 c}^{2}\left(d p_{1}\right)$.

3. Appendix. We organize some known results, occasionally with trivial variations, for easy reference.

I. Diffusion local time. All the basic facts we need are in [7]. As a reference for our purposes here the more leisurely [4] suffices and might be found more convenient.

(A) Brownian local time. Let $X=\left(X_{t}, \mathcal{C}_{t}, 0 \leq t<\infty,\left(P_{x}\right)\right)$ be coordinate representation of Brownian motion on function space $C$. The associated scale and speed are $x$ and $2 d x$.

Trotter's Theorem [14]. For each $t \geq 0$ and $x \in(-\infty, \infty)$ there exists a random variable $L(t, x ; X)$ such that for all $\omega$ in $C$ outside some fixed set $\Lambda$ with $P_{x}[\Lambda]=$ 0 for all $x$, the following two conditions bold: $(t, x) \rightarrow L(t, x ; X)(\omega)$ is continuous, and $\mu(t, B ; X)=\int_{B} L(t, x ; X) 2 d x, B$ a Borel set of $R^{1}, t \geq 0 . L(t, x ; X)$ is called Brownian local time.

(B) Ito-McKean representation. Let $P^{\prime}=\left(P_{x}^{\prime}\right), x \in I$, be a diffusion in $\mathscr{D}_{l}$, where $I$ is an open interval. Say $P^{\prime} \sim(p, m)$. A diffusion $Z$ corresponding to $P^{\prime}$ is constructed from Brownian motion $X$ in two steps. Let $P^{*} \sim\left(p^{*}, m^{*}\right)=$ $(p \circ q, m \circ q)$, where $q$ is the inverse function of $p . P^{*} \in \mathfrak{D}_{p(I)^{\bullet}}$. Then $Z^{*}=\left(Z_{t}^{*}\right)$ is obtained as $Z_{t}^{*}=X_{\beta(t)}, \beta(t)(=\beta(t, \omega))$ being the inverse of $\alpha(t)(=\alpha(t, \omega))$ defined by

$$
\alpha(t)=\int_{p(I)} L(t, y ; X) m^{*}(d y), \quad t<\tau=\inf \left\{s: X_{s} \notin p(I)\right\} .
$$

Finally $Z_{t}=q\left(Z_{t}^{*}\right)$. Note that as $t$ increases to $r, \alpha(t)$ approaches infinity. Both $\alpha(t)$ and $\beta(t)$ are continuous, strictly increasing. 
(C) Diffusion local time. Keeping the notations of $(A)$ and $(B), L(t, x ; Z)=$ $L(\beta(t), q(x) ; X)$ defines the local time of $Z$. Then $\mu(t, B ; Z)=\int_{B} L(t, x ; Z) m(d x)$, $B$ a Borel set of $I, 0 \leq t<\infty$, and $(t, x) \rightarrow L(t, x ; Z)$ is continuous, both assertions again holding outside the exceptional null set $\Lambda$. The last formula allows an obvious extension:

$$
\int_{0}^{t} f\left(Z_{u}\right) d u=\int_{I} L(t, x ; Z) f(x) m(d x), \quad f \text { Borel measurable, } \int|f| d m<\infty .
$$

So in particular, for the case of Brownian motion, $g \in L_{\text {loc }}^{2}$ is necessary and sufficient for $\int_{0}^{t} g^{2}\left(X_{u}\right) d u<\infty, P_{x}$-a.s. for all $t<\infty, x \in(-\infty, \infty)$.

(D) Change of time scale. We continue with the notations introduced. Let $n$ be a positive measure on $l$, finite on compact sets, assigning strictly positive weight to every open interval. Let $\gamma(t)=\int_{I} L(t, y ; Z) n(d y)$ and let $\delta(t)$ be the inverse function of $\gamma(t)$. The situation is similar to (B) above, but as $t$ tends to infinity $\gamma(t)$ tends to a limit $\gamma(\infty)$ which need not be infinite. So $\delta(t)$ is defined only for $0 \leq t<\gamma(\infty)$. The same considerations as in (B) show that $\left(Z_{\delta(t)}\right.$, $0 \leq t<\gamma(\infty))$ is a diffusion on $I$, defined up to the first exit time from $I$, and governed in the interior of $I$ by the scale $p$ and speed $n$.

II. Additive functionals of Brownian motion. Again $X=\left(X_{t}, C_{t}, 0 \leq t<\infty\right.$, $\left.\left(P_{x}\right)\right)$ is coordinate representation of Brownian motion, $\left(\theta_{t}\right)$ are the associated shift operators. A stochastic process $\left(A_{t}\right)$ is called an additive functional of Brownian motion if $A_{t}$ is $\mathcal{F}_{t}$-measurable, $A_{t}$ assumes values in $(-\infty, \infty]$ and, for each pair of nonnegative numbers $s, t, A_{t+s}=A_{s}+A_{t} \circ \theta_{s}, P_{x}-a_{. s},-\infty<x<\infty$. The following result is also given in Ventcel [15].

Tanaka's representation [13]. If $\left(A_{t}\right)$ is a finite-valued, continuous additive functional of Brownian motion, then there exists a continuous function $g$, and a function $k \in L_{\text {loc }}^{2}$ such that $A_{t}=g\left(X_{t}\right)-g\left(X_{0}\right)+\int_{0}^{t} k\left(X_{s}\right) d X_{s}$. We will require the following lemma.

Lemma. Let $\left(A_{t}\right)$ be an additive functional of Brownian motion with values in $(-\infty, \infty]$, continuous in the topology of the extended real line, with $A_{0}=0$

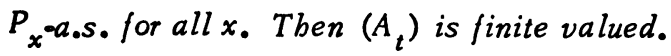

Proof. For every $x$ and every positive $\delta$ there exists a positive $\epsilon$ and a positive finite $M$ such that, with $\tau$ the first exit time of Brownian motion from $[x-\epsilon$, $x+\epsilon], P_{x}\left[\right.$ sup $\left._{0 \leq t \leq \tau}\left|A_{t}\right|>M\right]<\delta$. Now one can repeat, word for word, the argument of Tanaka [13, Theorem 1], to conclude that there exist positive constants $c$ and $\rho$, with $\rho<1$, such that

$$
P_{x}\left[\sup _{0 \leq t \leq \tau}\left|A_{t}\right|>\lambda\right] \leq c \rho^{\lambda}, \quad \lambda \geq 0 .
$$


In particular

$$
P_{x}\left[\sup _{0 \leq t \leq t}\left|A_{t}\right|<\infty\right]=1
$$

An easy covering argument concludes the proof.

III. Absolute continuity. Let $(\Omega, \mathcal{F})$ be a measurable space, $\left(\mathcal{F}_{t}\right)$ an increas-

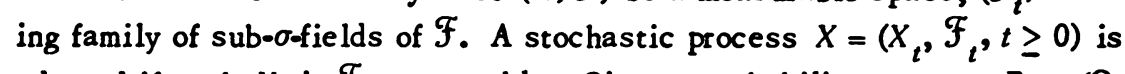
adapted if each $X_{t}$ is $\mathcal{F}_{t}$-measurable. Given a probability measure $P$ on $(\Omega, \mathcal{F})$, a continuous adapted process $X=\left(X_{t}, \mathcal{F}_{t}, t \geq 0\right)$ is said to be a Brownian motion under $P$ (the last phrase can be omitted if it is understood that a fixed $P$ is used) if $X$ is a martingale under $P$-measure with finite dimensional distributions as given by Wiener measure.

If $P$ is a probability measure on $\mathcal{F},\left.P\right|_{t}$ is the restriction of $P$ to $\mathcal{F}_{t}$. If $P^{\prime}$ is another such measure, with $\left.\left.P^{\prime}\right|_{t} \ll P\right|_{t}$ for each $t$, there exists a RadonNikodym derivative $L_{t}$ such that $P^{\prime}(\Lambda)=\int_{\Lambda} L_{t} d P, \Lambda \in \mathcal{F}_{t}$, and $\left(L_{t}, \mathcal{F}_{t}, t \geq 0\right)$ must be a nonnegative martingale with respect to $P$. We can choose a right continuous version. If $T_{0}=\inf \left\{t: L_{t}=0\right\} \leq \infty$, then $L_{t}=0$ for $t \geq T_{0}$. One verifies at once that if $S$ and $T$ are two bounded stopping times with $S \leq T$ and $H$ is an $\mathcal{F}_{T}$-measurable $P^{\prime}$-integrable random variable then

$$
E^{\prime}\left[H \mid \mathfrak{F}_{S}\right]=E\left[H\left(L_{T} / L_{S}\right) \mid F_{S}\right] \quad P^{\prime} \text {-a.s. }
$$

where the possible vanishing of $L_{S}$ causes no problem since $P^{\prime}\left[L_{S}=0\right]=0$.

Let $M=\left(M_{t}, \mathcal{F}_{t}, t \geq 0, P\right)$ be a continuous local martingale. Let $A_{t}=$ $\langle M, M\rangle_{t}$ be the associated increasing process, where we use the bracket notation of Meyer [12]. One defines a new process $X=\operatorname{Exp}[M]$ by $X_{t}=\exp \left[M_{t}-1 / 2 A_{t}\right]$. By Ito's formula this is again a continuous increasing process with $d X_{t}=X_{t} d M_{t}$. Since $X$ is in fact a positive continuous local martingale an easy limiting argument using Fatou's lemma shows it is a supermartingale. Evidently $X$ will be a martingale if and only if $E X_{t} \equiv 1$.

Conversely if $\left(Z_{t}\right)$ is a continuous adapted process such that $X_{t}=\exp \left[Z_{t}\right]$ is a continuous local martingale one sees, by applying Ito's formula to $\log X_{t}$, that $Z_{t}=M_{t}-1 / 2\langle M, M\rangle_{t}$ for some continuous local martingale $M$. In particular

$$
\begin{aligned}
& \left(M_{t}, \mathcal{F}_{t}, t \geq 0\right) \text { is Brownian motion if and only if } \\
& \left(\exp \left(M_{t}-1 / 2 t\right), \mathcal{F}_{t}, t \geq 0\right) \text { is a continuous local martingale. }
\end{aligned}
$$

It is also immediate that for two continuous local martingales $M$ and $N$

$$
\operatorname{Exp}[M+N]=\operatorname{Exp}[M] \cdot \operatorname{Exp}[N] \cdot \exp (-\langle M, N\rangle)
$$


Girsanov theorem [5]. Let $W=\left(W_{t}, \mathcal{F}_{t}, t \geq 0\right)$ be Brownian motion under $P$.

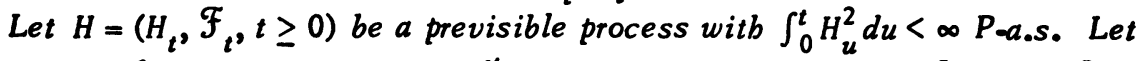
$V=W-\int_{0} H_{u} d u$ (i.e. $V_{t}=W_{t}-\int_{0}^{t} H_{u} d u, t \geq 0$ ) and set $L=\operatorname{Exp}\left[\int_{0} H_{u} d W_{u}\right]$.

If $L=\left(L_{t}, \mathcal{F}_{t}, t \geq 0\right)$ is a martingale and $P^{\prime}$ is determined by $P^{\prime}(\Lambda)=$ $\int_{\Lambda} L_{t} d P, \Lambda \in \mathcal{F}_{t}$, then with respect to $P^{\prime},\left(V_{t}, \mathcal{F}_{t}\right)$ is Brownian motion.

Proof. By (3.2) it must be proved that $\left(\exp \left(V_{t}-1 / 2 t\right), \mathcal{F}_{t}, t \geq 0\right)$ is a local martingale with respect to $P^{\prime}$. Writing out what this means, using (3.1) and (3.3) this follows at once.

Corollary (Kailath-Zakai [10]; with different proof Kadota-Shepp [8]). Let $W, H, V$ be as in the statement of Girsanov's theorem. (No bypotbesis on $L$ is made now.) Let $P^{0}$ and $P^{\prime}$ be the measures induced in function space $(C, \mathcal{C})$ by $W$ and $V$ respectively. Then $P_{t}^{\prime} \ll P_{t}^{0}$ for all $t$.

Proof. If $L$, defined as in Girsanov's the orem, is a martingale, the conclusion follows from Girsanov's theorem. In the general case there exist stopping times $T_{n} \uparrow \infty$ such that $L^{(n)}$, with $L_{t}^{(n)}=L_{t} \wedge T_{n}$, is a martingale for each $n$. Let $H_{t}^{(n)}=H_{t} \cdot X_{t \leq T_{n}}$. Then $L^{(n)}=\operatorname{Exp} H^{(n)}$ and, setting $V^{(n)}=W-\int_{0} H_{u}^{(n)} d u$, we find that the measures $P^{(n)}$ induced in function space by $V^{(n)}$ satisfy $P_{t}^{(n)} \ll$ $P_{t}^{0}$. Since for every $K \in \mathcal{C}_{t}, P^{(n)}(K)$ converges to $P^{\prime}(K)$ as $n$ goes to infinity $P_{t}^{\prime} \ll P_{t}^{0}$ follows.

The following is a variation of Dynkin [2, Theorem 10.4]. The notation $L_{t}[b]$ is defined in (1.2).

Transformation theorem. Let $\left(P_{x}\right)$ be a diffusion in $\mathscr{D}_{(-\infty, \infty)}, b \in L_{\text {loc }}^{1}$, and $E_{x}\left[L_{t}[b]\right] \equiv 1,-\infty<x<\infty$. Determine $P_{x}^{\prime}$ on $(C, \mathcal{C})$ by $P_{x}^{\prime}(\Lambda)=\int_{\Lambda} L_{t} d P, \Lambda \in \mathcal{C}_{t}$. Then $\left(P_{x}^{\prime}\right) \in \mathfrak{D}_{(-\infty, \infty)}$.

Proof. The existence of the $P_{x}^{\prime}$ is evident. In order to prove that $\left(P_{x}^{\prime}\right)$ is a strong Markov process consider a bounded Markov time $T$ (the unbounded case is handled by a limiting argument). Let $Y$ be a bounded, $C_{s}$-measurable random variable. Using (3.1), the fact that $\left(L_{t}\right)$ is a multiplicative functional, and the strong Markov property of $\left(P_{x}\right)$

$$
\begin{aligned}
E_{x}^{\prime}\left[Y \circ \theta_{T} \mid \mathcal{F}_{T}\right] & =E_{x}\left[Y \circ \theta_{T} \cdot\left(L_{T+s} / L_{T}\right) \mid F_{T}\right] \\
& =E_{x}\left[Y \circ \theta_{T} \cdot L_{s} \circ \theta_{T} \mid F_{T}\right]=E_{X_{T}}\left[L_{s} Y\right]=E_{X_{T}}^{\prime}[Y] .
\end{aligned}
$$

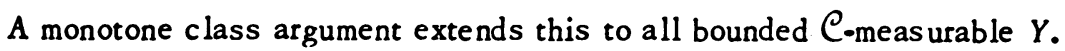

\section{BIBLIOGRAPHY}

1. L. Breiman, Probability, Addison-Wesley, Reading, Mass., 1968. MR 37 \#4841. 
2. E. B. Dynkin, Markov processes, Fizmatgiz, Moscow, 1963; English transl., Vols. I, II, Die Grundlehren der math. Wissenschaften, Bände 121, 122, Academic Press, New York; Springer-Verlag, Berlin, 1965. MR 33 \#1886; \#1887.

3. W. Feller, The parabolic differential equations and the associated semi-groups of transformations, Ann. of Math. (2) 55 (1952), 468-519. MR 13, 948.

4. D. Freedman, Brownian motion and diffusion, Holden-Day, San Francisco, Calif., 1971. MR 45 \#6074.

5. I. V. Girsanov, On transforming a class of stochastic processes by absolutely continuous substitution of measures, Teor. Verojatnost. i Primenen. 5 (1960), 314-330= Theor. Probability Appl. 5 (1960), 285-301. MR 24 \#A2986.

6. M. Hitsuda, Representations of Gaussian processes equivalent to a Wiener process, Osaka J. Math. 5 (1968), 299-312. MR 39 \#4935.

7. K. Ito and H. P. McKean, Jr., Diffusion processes and their sample paths, Die Grundlehren der math. Wissenschaften, Band 125, Academic Press, New York; SpringerVerlag, Berlin, 1965. MR 33 \#8031.

8. T. T. Kadota and L. A. Shepp, Conditions for absolute continuity between a certain pair of probability measures, Z. Wahrscheinlichkeitstheorie und Verw. Gebiete 16 (1970), 250-260. MR $43 \# 4074$.

9. T. Kailath, The structure of Radon-Nikodým derivatives with respect to Wiener and related meas ures, Ann. Math. Statist. 42 (1971), 1054-1067. MR 43 \#6997.

10. T. Kailath and M. Zakai, Absolute continuity and Radon-Nikod'́m derivatives for certain measures relative to Wiener measure, Ann. Math. Statist. 42 (1971), 130-140. MR 43 \#5608.

11. H. Kunita and S. Watanabe, On square integrable martingales, Nagoya Math. J. 30 (1967), 209-245. MR 36 \#945.

12. P. A. Meyer, Intégrales stochastiques. I, II, III, IV, Séminaire de Probabilités (Univ. Strasbourg, Strasbourg, 1966/67), Vol. I, Springer, Berlin, 1967, pp. 72-162. MR $37 \# 7000$.

13. H. Tanaka, Note on continuous additive functionals of the 1-dimensional Brownian path, Z. Wahrscheinlichkeitstheorie und Verw. Gebiete 1 (1962/63), 251-257. MR 29 \#6559.

14. H. F. Trotter, A property of Brownian motion paths, Illinois J. Math. 2 (1958), 425-433. MR 20 \#2795.

15. A. D. Ventcel', On continuous additive functionals of a multidimensional Wiener process, Dokl. Akad. Nauk SSSR 142 (1962), 1223-1226 = Soviet Math. Dokl. 3 (1962), 264-266. MR 27 \#265.

DE PAR TMENT OF MATHEMATICS, UNIVERSITY OF MINNESOTA, MINNEA POLIS, MINNE• SOTA 55455 